\title{
A YOUNG-PLANET SEARCH IN VISIBLE AND INFRARED LIGHT: DN TAURI, V836 TAURI, AND V827 TAURI ${ }^{1}$
}

\author{
L. Prato, ${ }^{2,3}$ M. Huerta, ${ }^{4}$ C. M. Johns-Krull ${ }^{3,5}$ N. Mahmud, ${ }^{3,5}$ D. T. Jaffe, ${ }^{6}$ and P. Hartigan ${ }^{5}$ \\ Received 2008 July 25; accepted 2008 September 17; published 2008 October 3
}

\begin{abstract}
In searches for low-mass companions to late-type stars, correlation between radial velocity variations and line bisector slope changes indicates contamination by large starspots. Two young stars demonstrate that this test is not sufficient to rule out starspots as a cause of radial velocity variations. As part of our survey for substellar companions to T Tauri stars, we identified the $\sim 2$ Myr old planet host candidates DN Tau and V836 Tau. In both cases, visible-light radial velocity modulation appears periodic and is uncorrelated with line bisector span variations, suggesting close companions of several $M_{\text {Jup }}$ in these systems. However, high-resolution, infrared spectroscopy shows that starspots cause the radial velocity variations. We also report unambiguous results for V827 Tau, identified as a spotted star on the basis of both visible-light and infrared spectroscopy. Our results suggest that infrared follow-up observations are critical for determining the source of radial velocity modulation in young, spotted stars.
\end{abstract}

Subject headings: planetary systems: formation — stars: individual (DN Tauri, V836 Tauri, V827 Tauri) — stars: spots — techniques: radial velocities

\section{INTRODUCTION}

Extrasolar planets are common; over 300 systems have been discovered. ${ }^{7}$ Recent studies have targeted higher mass (Sato et al. 2007; Johnson et al. 2008), lower mass (Butler et al. 2006; Endl et al. 2006), and younger objects (Paulson \& Yelda 2006; Setiawan et al. 2007). Identifying young planets is important to define the timescale for planet formation and thus distinguish the possible formation process(es).

Young stars still surrounded by the circumstellar material which forms planets are typically located at distances of $>100$ $\mathrm{pc}$ and are thus inherently faint and often obscured. They also manifest strong magnetic activity (e.g., Johns-Krull 2007) and are highly spotted. Numerous, large spots complicate detection of extrasolar planets through radial velocity (RV) monitoring (Saar \& Donahue 1997) because a spot that is partially visible at all times on the surface of an inclined star mimics RV modulation (e.g., Bouvier et al. 2007; Huerta et al. 2008).

Paulson \& Yelda (2006) studied 12-300 Myr old nearby stars and found no evidence for planets with masses $>1-2 M_{\text {Jup }}$ at the $3 \sigma$ level. Setiawan et al. (2007) identified a minimummass $6.1 M_{\text {Jup }}$ planet in a 852 day period orbit around the 100 Myr old G1-G1.5 V star HD 70573. More recently, Setiawan et al. (2008) reported a $\sim 10 M_{\text {Jup }}$ planet in a 3.56 day orbit around the $10 \mathrm{Myr}$ old star TW Hya, although Huelamo et al. (2008) identify this result as attributable to spots.

In this Letter we present our observations of the young stars

\footnotetext{
${ }^{1}$ This Letter includes data taken at the McDonald Observatory of the University of Texas at Austin.

${ }^{2}$ Lowell Observatory, 1400 West Mars Hill Road, Flagstaff, AZ 86001; lprato@lowell.edu.

${ }^{3}$ Visiting Astronomer at the Infrared Telescope Facility, which is operated by the University of Hawaii under cooperative agreement NCC 5-538 with the National Aeronautics and Space Administration, Office of Space Science, Planetary Astronomy Program.

${ }^{4}$ American Astronomical Society, 2000 Florida Avenue, Suite 400, Washington, DC 20009; marcos.huerta@ aas.org.

${ }_{5}^{5}$ Department of Physics and Astronomy, Rice University, MS-108, 6100 Main Street, Houston, TX 77005; cmj@rice.edu, naved@rice.edu, hartigan@ rice.edu.

${ }^{6}$ Department of Astronomy, University of Texas, R. L. Moore Hall, Austin, TX 78712; dtj@astro.as.utexas.edu.

${ }^{7}$ See, e.g., http://exoplanet.eu.
}

DN Tau, V836 Tau, and V827 Tau. While the V827 Tau visiblelight data clearly implicate spots as the cause of the apparent $\mathrm{RV}$ variability, corresponding data for DN Tau and V836 Tau suggest the presence of giant planets. Our infrared (IR) observations show that spots cause the RV variations seen in all three stars. In $\S 2$ we describe the observations, in $\S 3$ present our data analysis and the evidence for spots, and in $\S 4$ provide a brief discussion. We summarize in $\S 5$.

\section{OBSERVATIONS AND DATA REDUCTION}

\subsection{Visible-Light Spectroscopy}

Visible-light spectra of DN Tau (M0), V836 Tau (K7), and V827 Tau (K7) were taken at the McDonald Observatory 2.7 m Harlan J. Smith telescope, between 2004 November and 2008 January with the Coudé echelle spectrograph (Tull et al. 1995). A $1.2^{\prime \prime}$ slit yielded $R \sim 60,000$. Integration times were $\sim 1800$ $\mathrm{s}$; average seeing was $\sim 2^{\prime \prime}$. ThAr exposures taken immediately before and after each spectrum provided wavelength calibration; typical rms values for the dispersion solution precision were $\sim 4 \mathrm{~m} \mathrm{~s}^{-1}$. RV standards (Nidever et al. 2002; Butler et al. 1996; Cumming et al. 1999) were observed on every night of every run; their overall rms scatter is $140 \mathrm{~m} \mathrm{~s}^{-1}$. We obtained 43 spectra of DN Tau, 21 of V836 Tau, and 20 of V827 Tau and applied standard IRAF reduction routines. Details are given in Huerta (2007) and Huerta et al. (2008).

\subsection{Infrared Spectroscopy}

We observed DN Tau, V836 Tau, V827 Tau, and the RV standards HD 65277 (K5) and GJ 281 (M0) on UT 2008 February 13-20 with CSHELL (Tokunaga et al. 1990; Greene et al. 1993), the high-resolution, IR spectrograph at the NASA IRTF $3 \mathrm{~m}$ telescope. The seeing was $0.4^{\prime \prime}-0.8^{\prime \prime}$. Each object was observed on 6-8 nights. The $0.5^{\prime \prime}$ slit yielded $R \sim 46,000$. We obtained data in $10^{\prime \prime}$ nodded pairs. Spectra were centered at $2.298 \mu \mathrm{m}$ (vacuum). Integration times were $\sim 1 \mathrm{hr}$ for the $\mathrm{T}$ Tauri stars and $\sim 8$ minutes for the standards. The signal-tonoise ratio $(\mathrm{S} / \mathrm{N})$ was $\sim 70-120$. Data were reduced as described in Johns-Krull et al. (1999). 

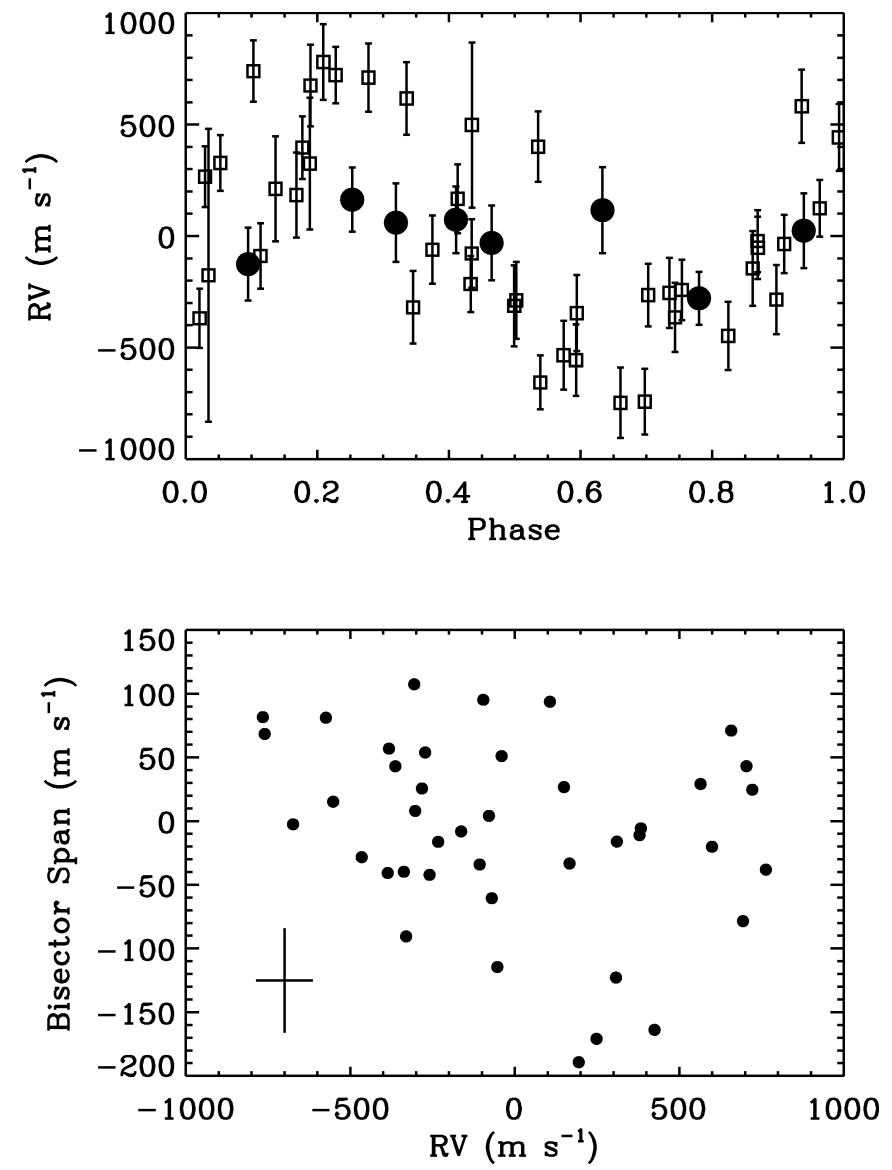

FIG. 1.-Top: Relative RV vs. phase for DN Tau. Visible-light (open squares) and IR (filled circles) data have been phased to a period of $6.33 \pm$ 0.20 days. Bottom: Line bisector spans as a function of RV for the visiblelight data. The linear correlation coefficient is $r=-0.27$ and the associated FAP, $f_{p}$, is 0.09 .

\section{ANALYSIS}

\subsection{Radial Velocities: Visible Light}

Relative RVs were determined by cross-correlating a high$\mathrm{S} / \mathrm{N}$, fiducial spectrum against all other spectra for the same target. We used 6 orders spanning $~ 5700$ to $6800 \AA$ A. Uncertainties were estimated from the standard deviation of the mean for the 6 orders, added in quadrature with the $140 \mathrm{~m} \mathrm{~s}^{-1}$ uncertainty derived from the RV standards $(\S 2$; Huerta et al. 2008). RVs were corrected for the Earth's barycentric motion.

Optimum periods and uncertainties for phasing the RV data were selected based on power spectra (Huerta et al. 2008). For DN Tau we found $P=6.33 \pm 0.20$ days and a false alarm probability (FAP) of $<0.001$; for V836 Tau, $P=2.48 \pm$ 0.49 days and FAP $=0.10$; and for V827 Tau, $P=3.76 \pm$ 0.06 days and FAP $<0.001$. We also checked for periodicity using the discrete Fourier transform plus CLEAN method of Roberts et al. (1987). The strongest power spectrum peaks for DN Tau and V836 Tau occur at the same periods. The CLEAN method recovered a best period of 3.61 days for V827 Tau, within $\sim 2 \sigma$ of the above estimate. The phased RV data are shown in Figures 1-3.

\subsection{Line Bisector Spans}

The presence of a starspot will distort the line profile at the $\mathrm{RV}$ that corresponds to the stellar velocity at the location of the spot. This distortion is in proportion to the ratio of quiescent
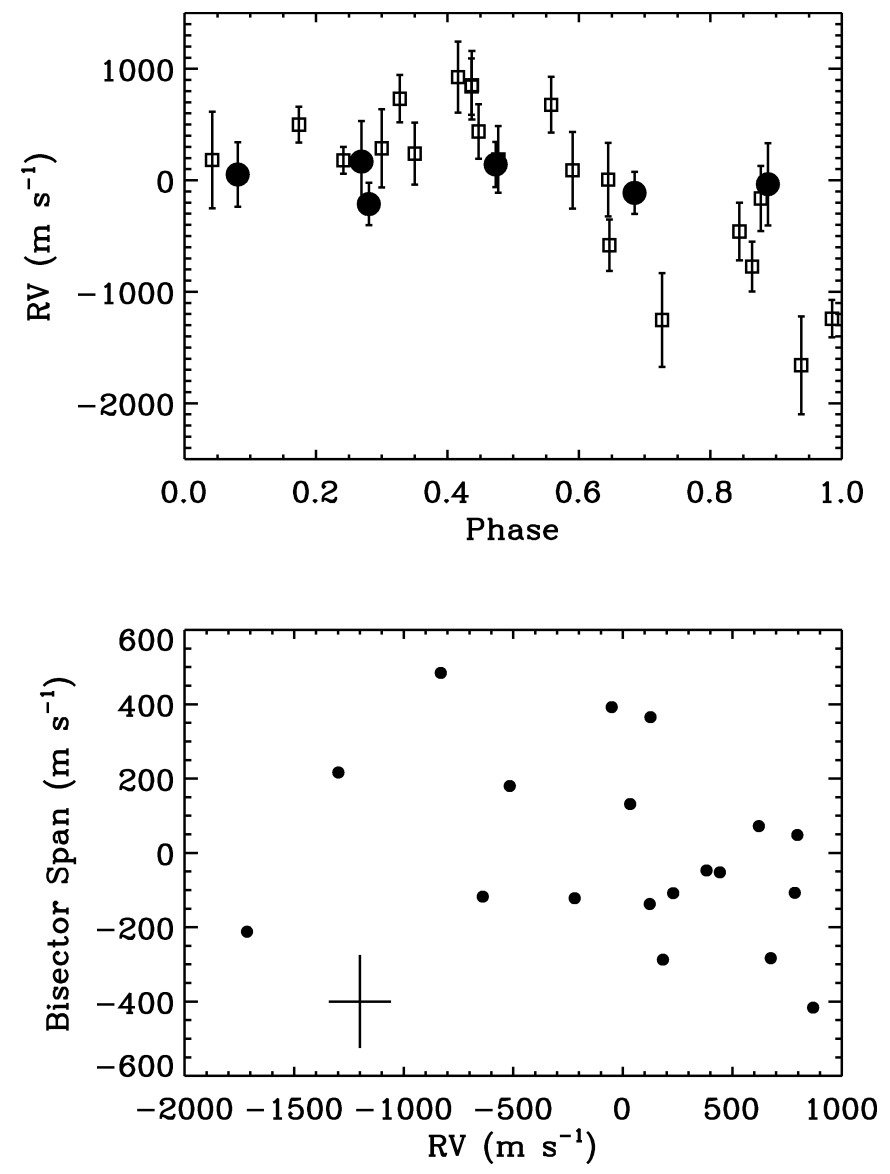

FIG. 2.-Same as Fig. 1 for V836 Tau, phased to a period of $2.48 \pm 0.49$ days $\left(r=-0.30, f_{p}=0.20\right)$.

photosphere surface brightness and surface brightness within the spot, at the observing wavelength, and the fraction of stellar surface covered by the spot (e.g., Queloz et al. 2001). Thus, asymmetries in the line profiles originating from spots will be present for all lines in the spectrum of a young star and are typically correlated with the RV measured from the same spectrum. These asymmetries have become the standard criterion for rejecting starspots as the cause of false RV signals (e.g., Queloz et al. 2001; Bouvier et al. 2007; Huerta et al. 2008; Setiawan et al. 2008). For each of the 6 orders used to determine the RVs, we cross-correlated all absorption lines and measured the cross-correlation function (CCF) for that order. The average of these six CCFs was used to measure the bisector spans (bottom panels of Figs. 1-3). The linear correlation coefficient and associated FAP (Bevington \& Robinson 1992) is listed in the captions. As expected for a spotted star, a clear correlation between bisector span and RV is observed for V827 Tau. DN Tau and V836 Tau show no correlation, suggesting that the variability is not the result of spots.

\subsection{Infrared Radial Velocity Signatures}

The contrast between a $4000 \mathrm{~K}$ photosphere and a $3000 \mathrm{~K}$ spot (Bouvier \& Bertout 1989) is greater in visible than in IR light because flux scales as a steeper function of temperature at wavelengths shorter than the blackbody peak (e.g., Carpenter et al. 2001). Given the decreased spot to photosphere contrast in the near-IR, the amplitude of the RV modulation will be smaller. Conversely, if a planet drives the RV modulation, the amplitude should be the same in visible and IR light. 

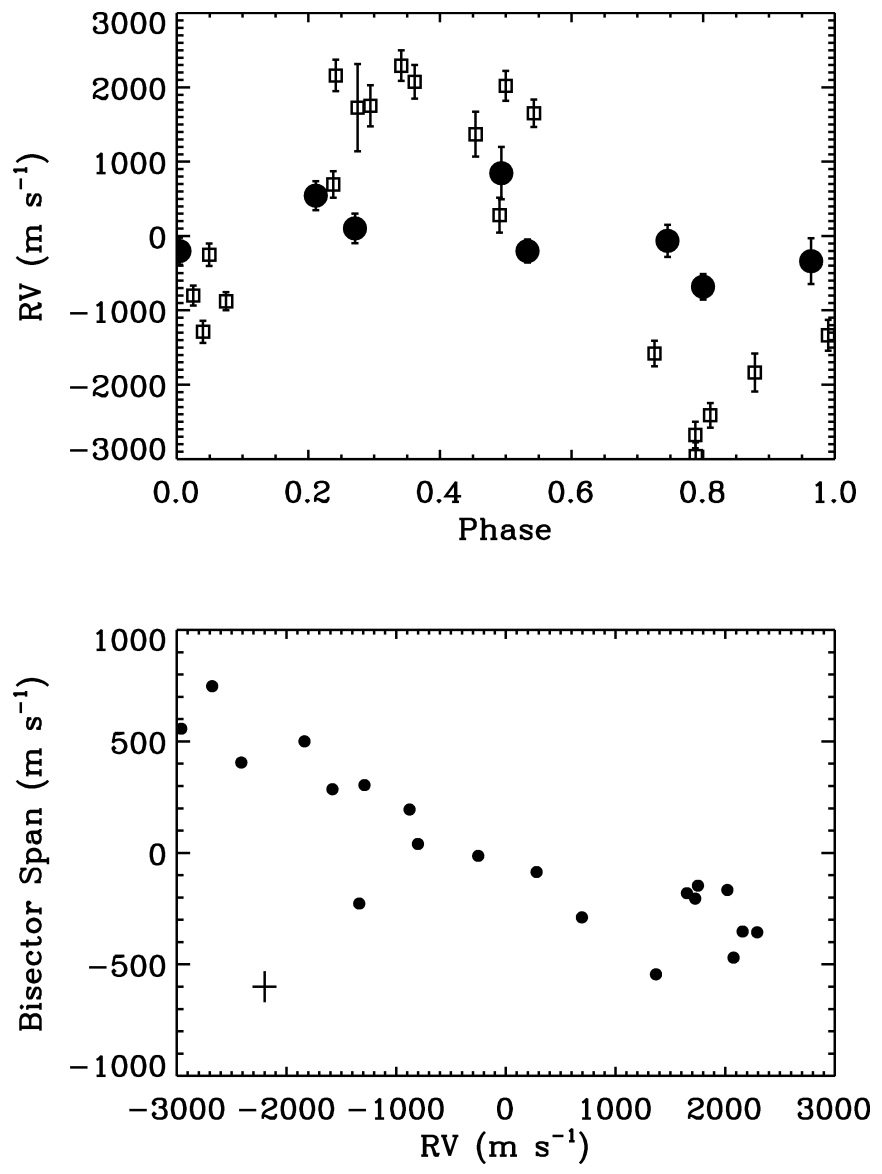

Fig. 3.-Same as Fig. 1 for V827 Tau, phased to a period of $3.76 \pm 0.06$ days $\left(r=-0.88, f_{p}=2.8 \times 10^{-7}\right)$.

Blake et al. $(2007,2008)$ used near-IR observations to search for companions to low-mass objects, exploiting telluric absorption lines for high-precision RV measurements. Figure 4 shows an example of a GJ $281 \mathrm{~K}$-band spectrum and illustrates our similar approach. We created models by combining highresolution telluric absorption (Livingston \& Wallace 1991) and cool stellar spectra (the sunspot atlas of Wallace \& Livingston 1992), applying a range of velocity shifts relative to the telluric lines. Other free parameters are $v \sin i$, a Gaussian FWHM for the spectrometer line-spread function, scale factors for line depths, and a first-order continuum normalization function. We employed the Marquardt method for nonlinear least-squares fitting (Bevington \& Robinson 1992) of each model to an observed spectrum. The difference between the stellar and telluric velocities in the best-fit model spectrum yields the RV, which was then corrected for barycentric motion.

Figures 1-3 show the IR-derived relative RVs. The data are phased to the periods given in $\S 3.1$. The RV standard deviation in the IR data for HD 65277 is $127 \mathrm{~m} \mathrm{~s}^{-1}$ and for GJ 281 is $98 \mathrm{~m} \mathrm{~s}^{-1}$. Internal errors, measured from the least-squares fitting, are $\sim 40 \mathrm{~m} \mathrm{~s}^{-1}$ for both standards. For the young stars, internal errors were $100-300 \mathrm{~m} \mathrm{~s}^{-1}$, depending on the $\mathrm{S} / \mathrm{N}$ achieved. Random errors, which we assume add in quadrature with our internal errors to give the overall scatter in velocities, are $120 \mathrm{~m} \mathrm{~s}^{-1}$ for HD 65277 and $90 \mathrm{~m} \mathrm{~s}^{-1}$ for GJ 281. We use $110 \mathrm{~m} \mathrm{~s}^{-1}$ as our final value for the random errors. The uncertainties shown for the IR data in Figures 1-3 represent the sum, in quadrature, of the individual internal error and the 110 $\mathrm{m} \mathrm{s}^{-1}$ random error. Within our measurement precision, we are

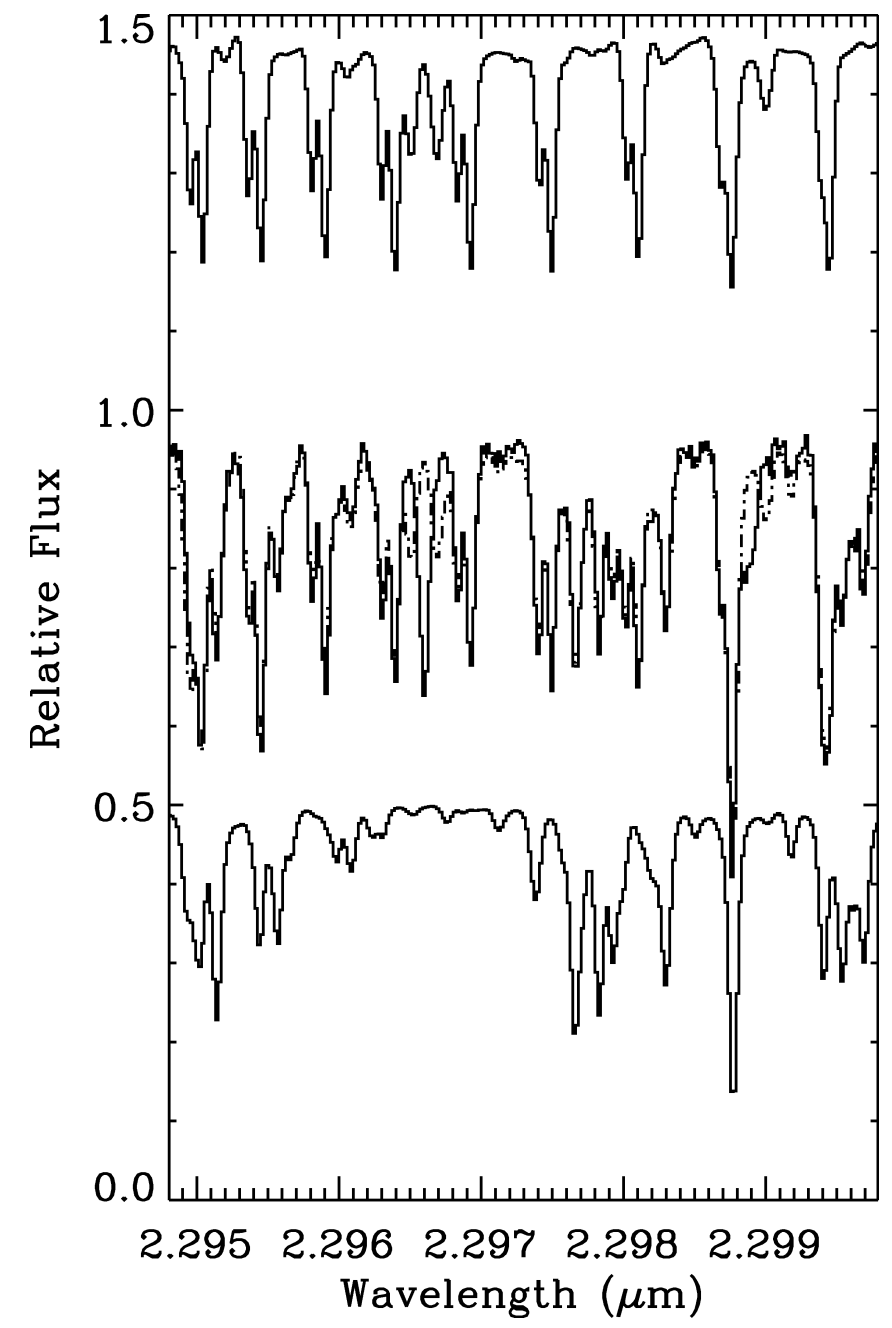

FIG. 4.-Middle: UT 2008 February 20 CSHELL spectrum (solid line) of the RV standard GJ 281 and a model (dash-dotted line) consisting of a stellar photosphere component (top: from the NSO sunspot atlas) and a telluric component (bottom: from the NOAO spectral atlas).

unable to detect any IR RV variability in DN Tau and V836 Tau. V827 Tau shows significant IR RV variations but at a reduced amplitude from those observed in visible light.

\section{DISCUSSION}

Figures 1 and 2 show the visible-light RV modulation for DN Tau and V836 Tau, with full amplitudes of $\sim 1500$ and $\sim 2700 \mathrm{~m} \mathrm{~s}^{-1}$, respectively. Within the $1 \sigma$ uncertainties, all but one point in the IR RVs of DN Tau are consistent with zero; all six IR RVs of V836 Tau also show no variation. These results indicate that no planets are present around DN Tau or V836 Tau with masses greater than a few $M_{\text {Jup }}$ at $<0.5 \mathrm{AU}$ or $\sim 10 M_{\text {Jup }}$ at $\sim 1 \mathrm{AU}$, despite the absence of a correlation between the visible-light RVs and bisector spans. Mathieu et al. (1989) identify V836 Tau and V827 Tau as RV variables with peakto-peak amplitudes of 7-8 $\mathrm{km} \mathrm{s}^{-1}$. Apparently the density and size of spots on V836 Tau vary; historical data may serve as an additional criterion for heavily spotted young stars.

The primary conclusion from our visible-light data is that the lack of a correlation between the line bisector span and the $\mathrm{RV}$ is not proof of a reflex motion companion. In addition, the $\mathrm{RV}$ period can change significantly with new data. Initial analysis of 20 visible-light RVs for DN Tau, taken over 2.5 years, 
indicated convincing modulation with $P=7.5$ days and FAP $=0.002$. Although not markedly different from rotation period estimates of 6.0-6.4 days (Bouvier et al. 1993; Percy et al. 2006), $\chi^{2}$ for $P=7.5$ days was more favorable than that for a secondary peak at 6.3 days. Removing the fit to either the 7.5 or 6.3 day period from the RV data and recalculating the power spectrum yielded no significant peak, suggesting that the data were best represented with only one period. With the addition of RVs measured in winter 2007-2008, the integrity of the 7.5 day period was diminished and the 6.3 day period came to dominate the power spectrum. The rotation period of V836 Tau has been stable for decades at 6.76 days (Grankin et al. 2008). We find a different RV period, 2.48 days, and substantially reduced power in the RV modulation near 6.76 days. The RV period for V827 Tau, 3.76 days, is indistinguishable from previously determined values of the rotation period (Grankin et al. 2008).

If the visible-light RV modulation originates in spots, why are the bisector spans not correlated (Figs. 1 and 2)? Figure 3 clearly shows correlation for V827 Tau; many other stars show a correlation as well (e.g., Queloz et al. 2001; Bouvier et al. 2007; Huerta et al. 2008). We do not believe that this can be attributed to a stronger impact of spots on particular spectral lines because the same echelle orders were used to determine the bisector spans for all targets. Desort et al. (2007) show that when $v \sin i$ is smaller than the spectrometer resolution, RV and bisector variations originating in spots can mimic the behavior expected from short-period giant planets. The $v \sin i$ of DN Tau and V836 Tau, $10 \mathrm{~km} \mathrm{~s}^{-1}$, are not much larger than our visible-light spectral resolution, $5 \mathrm{~km} \mathrm{~s}^{-1}$, while V827 Tau and LkCa 19 (Huerta et al. 2008) have $v \sin i$ values of $\sim 20$ $\mathrm{km} \mathrm{s}^{-1}$, suggesting qualitative agreement with the simulations of Desort et al. (2007).

\section{SUMMARY}

We have measured the RVs of the 2 Myr old T Tauri stars DN Tau, V836 Tau, and V827 Tau in visible and IR light; the variations we see in all three systems are likely the result of starspots. Furthermore: (1) Periodogram analysis can reveal the presence of a period but not necessarily a reliable value until RV measurements densely sample the full phase of the periodic signal. (2) The lack of correlation of line bisector spans with RVs for some stars with spots is an important problem to address in the search for planets around young stars. (3) Highresolution, IR spectroscopy is critical for the verification of young planet candidates; without the contrast in RV modulation amplitude between the visible-light and IR data, it is unclear whether spots or a companion cause variability. (4) In the case of the spotted young star, V827 Tau (Fig. 3), we observe a substantial reduction in RV amplitude between the visible-light and IR data. This result can be exploited to improve our understanding of starspot temperature and filling factors. (5) $\mathrm{T}$ Tauri stars are virtually guaranteed to have spots at some level; if planets are also present, their detection will likely require disentanglement of the blended RV signals and will benefit greatly from measurements that span a broad range in wavelength.

The authors acknowledge the SIM Young Planets Key Project (PI C. Beichman) for research support; funding was also provided by NASA grant 05-SSO05-86. We thank G. Blake, C. Salyk, and E. Schaller for sharing Keck time (our first attempt at IR observations of DN Tau), D. Gies and A. Fullerton for their assistance implementing the CLEAN power spectrum estimate, and the referee, J. Eisloeffel, for a prompt and helpful report. L. P. thanks P. Bodenheimer and T. Barman for informative discussions. This work made use of the SIMBAD database, the NASA Astrophysics Data System, and the Two Micron All Sky Survey, a joint project of the University of Massachusetts and IPAC/Caltech, funded by NASA and the NSF. We recognize the significant cultural role that Mauna Kea plays in the indigenous Hawaiian community and are grateful for the opportunity to observe there.

\section{REFERENCES}

Bevington, P. R., \& Robinson, D. K. 1992, Data Reduction and Error Analysis for the Physical Sciences (2nd ed.; New York: McGraw-Hill)

Blake, C. H., Charbonneau, D., White, R. J., Marley, M. S., \& Saumon, D. 2007, ApJ, 666, 1198

Blake, C. H., Charbonneau, D., White, R. J., Torres, G., Marley, M. S., \& Saumon, D. 2008, ApJ, 678, L125

Bouvier, J., \& Bertout, C. 1989, A\&A, 211, 99

Bouvier, J., Cabrit, S., Fernandez, M., Martin, E. L., \& Matthews, J. M. 1993, A\&A, 272, 176

Bouvier, J., et al. 2007, A\&A, 463, 1017

Butler, R. P., Johnson, J. A., Marcy, G. W., Wright, J. T., Vogt, S. S., \& Fischer, D. A. 2006, PASP, 118, 1685

Butler, R. P., Marcy, G. W., Williams, E., McCarthy, C., Dosanjh, P., \& Vogt, S. S. 1996, PASP, 108, 500

Carpenter, J. M., Hillenbrand, L. A., \& Skrutskie, M. F. 2001, AJ, 121, 3160

Cumming, A., Marcy, G. W., \& Butler, R. P. 1999, ApJ, 526, 890

Desort, M., Lagrange, A.-M., Galland, F., Udry, S., \& Mayor, M. 2007, A\&A, 473, 983

Endl, M., Cochran, W. D., Kürster, M., Paulson, D. B., Wittenmyer, R. A., MacQueen, P. J., \& Tull, R. G. 2006, ApJ, 649, 436

Grankin, K. N., Bouvier, J., Herbst, W., \& Melnikov, S. Y. 2008, A\&A, 479, 827

Greene, T. P., Tokunaga, A. T., Toomey, D. W., \& Carr, J. B. 1993, Proc. SPIE, 1946, 313

Huelamo, N., et al. 2008, A\&A, in press

Huerta, M. 2007, Ph.D. thesis, Rice University
Huerta, M., Johns-Krull, C. M., Prato, L., Hartigan, P., \& Jaffe, D. T. 2008, ApJ, 678, 472

Johns-Krull, C. M. 2007, ApJ, 664, 975

Johns-Krull, C. M., Valenti, J. A., \& Koresko, C. 1999, ApJ, 516, 900

Johnson, J. A., et al. 2008, ApJ, 675, 784

Livingston, W., \& Wallace, L. 1991, Atlas of the Solar Spectrum in the Infrared from 1850 to $9000 \mathrm{~cm}^{-1}$ (NSO Tech. Rep.; Tucson: NSO)

Mathieu, R. D., Walter, F. M., \& Myers, P. C. 1989, AJ, 98, 987

Nidever, D. L., Marcy, G. W., Butler, R. P., Fischer, D. A., \& Vogt, S. S. 2002, ApJS, 141, 503

Paulson, D. B., \& Yelda, S. 2006, PASP, 118, 706

Percy, J. R., Gryc, W. K., Wong, J. C.-Y., \& Herbst, W. 2006, PASP, 118, 1390

Queloz, D., et al. 2001, A\&A, 379, 279

Roberts, D. H., Lehar, J., \& Dreher, J. W. 1987, AJ, 93, 968

Saar, S. H., \& Donahue, R. A. 1997, ApJ, 485, 319

Sato, B., et al. 2007, ApJ, 661, 527

Setiawan, J., Henning, T., Launhardt, R., Müller, A., Weise, P., \& Kürster, M. 2008, Nature, 451, 38

Setiawan, J., Weise, P., Henning, Th., Launhardt, R., Müller, A., \& Rodmann, J. 2007, ApJ, 660, L145

Tokunaga, A. T., Toomey, D. W., Carr, J., Hall, D. N. B., \& Epps, H. W. 1990, Proc. SPIE, 1235, 131

Tull, R. G., MacQueen, P. J., Sneden, C., \& Lambert, D. L. 1995, PASP, 107, 251

Wallace, L., \& Livingston, W. C. 1992, Atlas of a Dark Sunspot Umbral Spectrum from 1970 to $8640 \mathrm{~cm}^{-1}$ (NSO Tech. Rep. 92-001; Tucson: NSO) 